\title{
ANALYSIS OF ADVANCED TECHNIQUES OF IMAGE PROCESSING BASED ON AUTOMATIC DETECTION SYSTEM AND ROAD SIGNS RECOGNITION
}

\author{
Dorota Smorawa, Mariusz Kubanek \\ Institute of Computer and Information Sciences, Czestochowa University of Technology \\ Częstochowa, Poland \\ dorota.smorawa@icis.pcz.pl,mariusz.kubanek@icis.pcz.pl
}

\begin{abstract}
This paper describes implementations of the Hough Circle Transform and the Radon Line Transform. The presented transforms were used to build a system for identifying road signs from selected images and recordings. This can be very important and especially useful for monitoring and prevention in driver assistance systems. The design of our system assumed that the objects should be found automatically from images or video sequence. Furthermore, the detection was based on the shapes of common traffic signs which correspond to used transforms. Additionally, the Huffman method was applied to encode the data before being compared. Consequently, the traffic signs were matched directly with the Euclidean distance.
\end{abstract}

Keywords: image processing, image detection, Radon transform, Hough transform

\section{Introduction}

A few years ago the term "safe car" was being used to describe a road vehicle that was equipped with a seat belt and stretchers. Then airbags were introduced and later also the ABS (Anti-lock braking system). Today, the automotive industry is developing at an impressive rate, especially in the field of safety and comfortable driving. The element of this development is, without doubt, an advanced car system, which improves driving comfort and increases our safety as well as the safety of other road users. An example of a system that affects the safety when driving a car can be the detection system which detects objects in the way such as people, animals or other elements. Other systems may include a parking assistant or even systems that prevent uncontrolled changing of lanes, which plays a significant role during long travel when a driver can fall asleep.

In this article a system which recognizes road signs from static images is presented. The system can distinguish signs based on their shape for instance: triangular, square or round. Various techniques and methods of image processing were used to design the system [1]. 


\section{The outline of detection and recognition of road signs methods}

One of the most important tasks when discussing advanced image processing is object detection and recognition. In order to determine what is in the image it is required to define what objects are there and where they are located. Usually object detection is connected with the preliminary recognition. Generally the first step in image recognition is edge detection, which allows one to identify the location of the objects. One of the most popular methods used for edge detection is the Canny edge detector which must satisfy three requirements:

- detection - algorithm should detect as many real edges as possible,

- localization - detected edges should be located maximally close to the real ones,

- minimal response - edges should be marked once and the noise should not create false edges.

The system of road signs recognition operates in two processes: sign registration and sign identification. The registration process handles the data acquisition and database management of the results. The marked new image of a road sign in the first stage is subjected to detection which allows efficient features extraction, so that later the sign can be encoded as numerical data and saved to the database correctly. The registration process is shown in Figure 1.

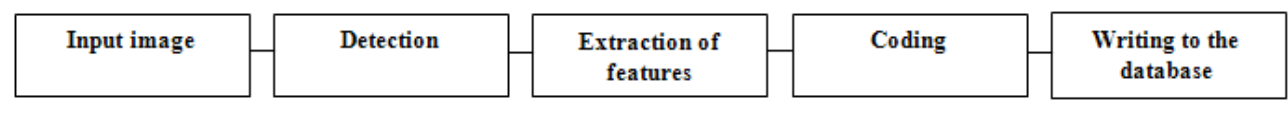

\section{The registration process}

Fig. 1. The process of road signs registration to the database

Once the sign has been registered correctly and the results have been stored in the database the process of identification is applied. At this stage the sign's designated characteristics are being compared with the earlier marked standard, which allows for the identification of a particular road sign. The process of identification is presented in Figure 2.

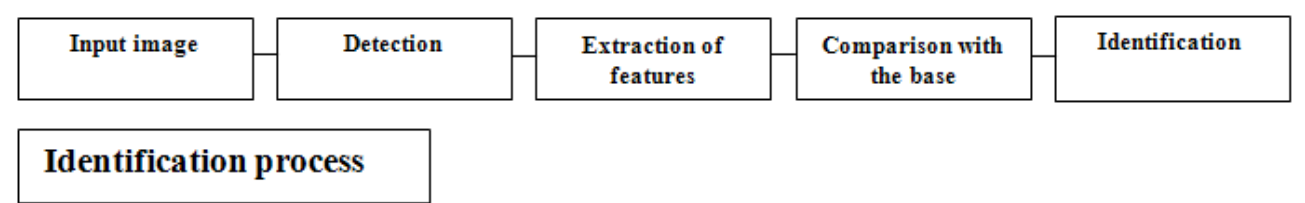

Fig. 2. The process of identifying road signs in the system

In the automotive industry there are already many systems which facilitate and improve the driving of a car $[1,2]$. An example of such a system may be the "Night Vision" system, which uses thermographic cameras and infrared light. The system is able to detect and recognize people, animals and other objects located in front of 
the vehicle, before they even become visible to a human eye. Moreover, the system allows one to see about 300 metres ahead, which not only helps the driver but also cares for his or her safety, as well as safety of other traffic participants. Another example is "Around View Monitor" system, also called a parking assistant. It uses four wide-angle cameras: one in the front, one at the back and two on the sides of a car, which provide a 360 degree view displayed on a screen. Another example of a driver safety system is "LDP" system, known as a system preventing accidental lane departure. The system is equipped with a camera mounted in a rear mirror casing. Images from the camera are sent to a computer which analyses them systematically and checks if the vehicle is still moving between the lines on the road. If the driver does not switch the indicator and the car crosses the line of the lane then the system reacts. A warning light appears on the dashboard and a distinctive sound is generated on the side of a vehicle drifting out of a lane. At the same time the system activates the LKS system - Lane Keeping System which automatically takes steps to ensure that the car stays in its lane. The system can also work at night and recognize white and yellow, continuous and interrupted road markings. LDP was created mainly for driving on highway and long straight roads. It does not work in cities or on crowded streets and below the speed of $70 \mathrm{~km} / \mathrm{h}[3-5]$.

\section{The methods used in the preliminary processing of an image}

Images are a special form of data and their specification has not been fully explored. However, digital image processing has been gaining more popularity in recent years. Vision systems appear in everyday life, starting from monitoring systems to biometric passports. Thanks to the use of appropriate image processing operations information which is usually not visible to a human visual system can be obtained.

The considerable part of digital image processing aim comes down to determine:

- what objects are in view,

- location of the objects on the image,

- why objects are in a given position.

The created vision system is used in spatial intelligence by means of a video converter (camera).The initial image processing is connected with different types of image filtering or brightness changes.

The first step that is performed after loading the image to the system is initial localization of the searched object based on thresholding algorithms and image noise reduction algorithm. A specified RGB value range, based on a three-dimensional histogram created from three colour-making attributes, has been assigned to each road sign. The application used multilevel threshold whose relative level threshold values are elements of the vector:

$$
T=\left[T_{1}, T_{2}, \ldots T_{N}\right]
$$


As a result the image was subject to segmentation in areas into $N+1$ with different brightness, for example:

$$
R 1: \text { if } f(x, y) \leq T_{1}, R 2 \text { : if } T_{1}<f(x, y), R 3: \text { if } f(x, y)>T_{2}
$$

The threshold value range has been determined based on static photos of road signs taken on a sunny, rainy or overcast day and at night. Parallel with a brightness threshold algorithm on the input image, after the edge detection has been performed, the Radon and Hough transforms follow to conduct alternative initial localization of road signs.

\subsection{Implementation of the Radon transform}

The Radon transform is a mathematical description of the projection process. The projection function can be presented in the following formula:

$$
P_{\phi}(t)=\int_{-\infty}^{\infty} \int_{-\infty}^{\infty} f(x, y) \delta(x \cos \phi+y \sin \phi-t) d x d y
$$

where:

$f(x, y)$ - function which describes spatial distribution of the projection lines,

$\delta \quad$ - Dirac delta distribution,

$\phi \quad$ - illumination angle,

$t \quad$ - shift with respect to the center,

$x \cos \phi, y \sin \phi$-coordinates of the points transferred to projection's system of coordinates,

$P_{\phi}(t)$ - projection function value with an angle $\phi$ and the shift $t$.

In transform the line's angle of inclination is $\phi$ to axis OY, its distance from the beginning of the system of coordinates is $t$. If the Radon transform is designated with a fixed angle $\phi$ with $t \in\left(t_{\min } ; t_{\max }\right)$, where $t_{\min }$ and $t_{\max }$ are matched so they can cover all the image, the image projection towards $\phi$ is obtained. In image recognition methods the Radon transform is used to detect lines which indicate shapes of a given object. The transform pair of points whose coordinates are $(x, y)$ a pair of $(\phi, t)$ is assigned. These parameters signify:

$t$-distance of the point in Cartesian space from beginning of the system of coordinates,

$\phi$ - vector distance angle of inclination to OX axis in the Cartesian space.

After the transform has been applied on the image a map of coefficients of the Radon transform is obtained, that shows the importance of coefficients in creating the image of the inverse of the Radon transform. Figure 3 shows image after edge detection and map of the Radon transform coefficients. 

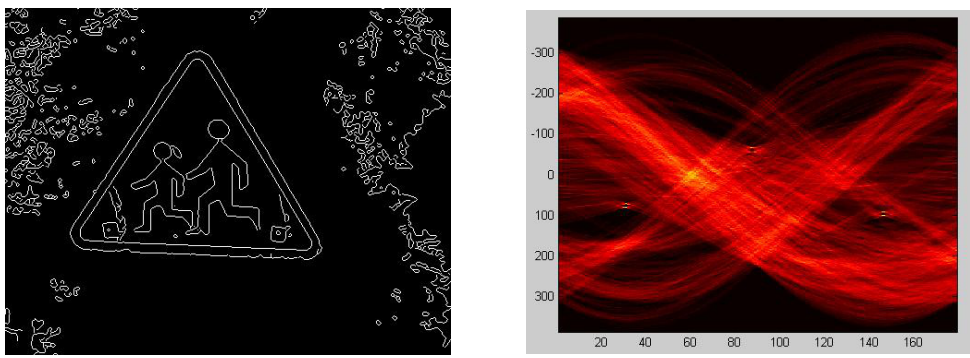

Fig. 3. Image after edge detection (left) and map of the Radon transform coefficients (right)

The map of coefficients must be transformed into a form understandable for people. The following formula helps reconstruct the projection lines function, also known as image reconstruction. The inverse Radon transform is defined by the following formula:

$$
f(x, y)=R^{-1}\left(P_{\phi}(t)\right)=\frac{1}{2 \pi^{2}} \int_{0}^{\pi} \int_{-\infty}^{\infty} \frac{\frac{\partial\left(P_{\phi}(t)\right)}{\partial t}}{(x \cos \phi+y \sin \phi-t)} d t d \phi
$$

where:

$f(x, y)$ - the function representing the distribution of the lines of projection, $R^{-1} \quad$ - inverse Radon transform,

$\partial / \partial t$ - direction derivative along the variable $t[6]$.

The inverse Radon transform is presented in Figure 4.

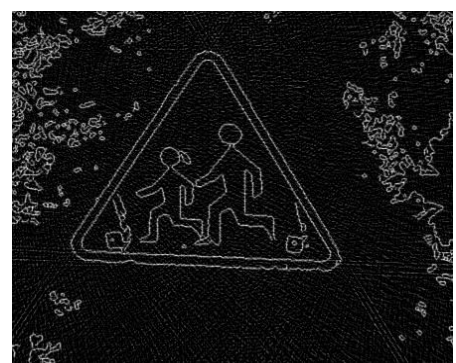

Fig. 4. The inverse Radon transform

\subsection{Implementation of the Hough transform}

The Hough transform's task is to localize the round road signs in the application. Similarly to the previous transform, this one is performed on the edgedetected image. In the Hough transform the point in Cartesian space assigned is simple or circle of a given radius and center in the point XY. The transform is described with the formula of circle equation:

$$
(x-a)^{2}+(y-b)^{2}=r^{2}
$$


where:

$x, y$ - axes of the coordinate system,

$r$ - radius length,

$a, b$ - coordinates of the center.

The idea of the algorithm:

- point on the image is fragment of a circle, so the parameters $x$ and $y$ are known,

- we assume that the centre of the circle may be found anywhere on the image,

- we know the length of the radius $r$.

The Hough transform is based on the transformation of the image into appropriate space and identifying the curve intersection parameters [7, 8]. Figures 5 and 6 show image after edge detection and Hough transform coefficients map and designated center of the circle on the basis of the underlying districts Hough Transform.
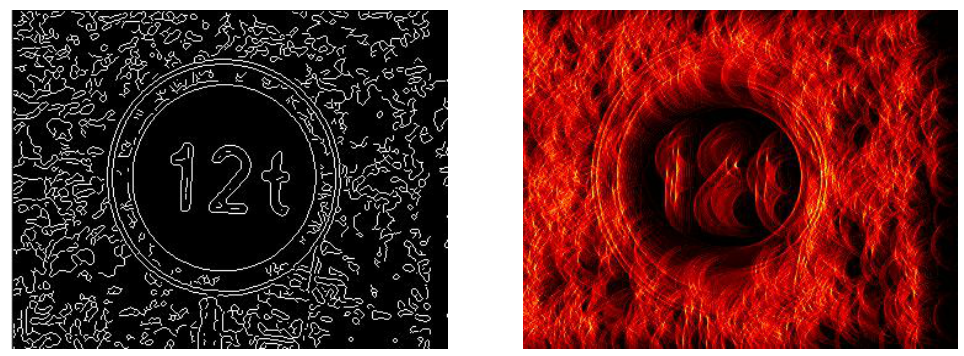

Fig. 5. Image after edge detection and Hough transform coefficients map

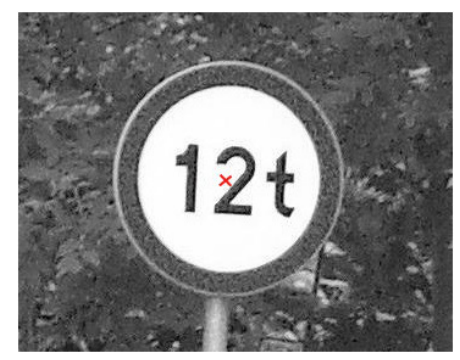

Fig. 6. Designated center of the circle on the basis of the underlying districts Hough Transform

\section{Extraction and coding characteristic features the image}

After initial location of the objects on the image and threshold algorithm, the segmentation of the image is applied by means of algorithms which determine limits and contours of the object on the image. In result we get a binary object which then is submitted to edge features extraction.

The features of the objects on the image being considered during the extraction can be divided into a few categories:

- edge features of the object, for example: line shape, 
- features of space object, for example: colour, type of texture, space,

- topological features of the shape.

In the created application the features extraction is based on the features of the edges of the object, as well as the space features. After the characteristic features have been extracted and the standard has been matched, the next step is coding features of the object by means of Huffman coding which, thanks to its construction, is widely used in lossless data compression.

The Huffman coding is based on the estimated probability of symbol occurrence in the input data stream. The main assumption of the method is to limit the code length for a particular symbol. The basis in the method is building a tree - Huffman's tree (see Fig. 7). The tree is built from the leaf to the root. Every leaf represents a single symbol from the data field. The value of a given leaf describes the symbol occurrence number in the input stream. In Huffman's tree there is only one way to each sign. If the links to the right side are marked 0 , and to the left side 1 it means that each sign (symbol) can be assigned with a unique binary code. For a given sequence of data more than one tree can be built which means that during decompression the tree according to which the data were encoded will be necessary.

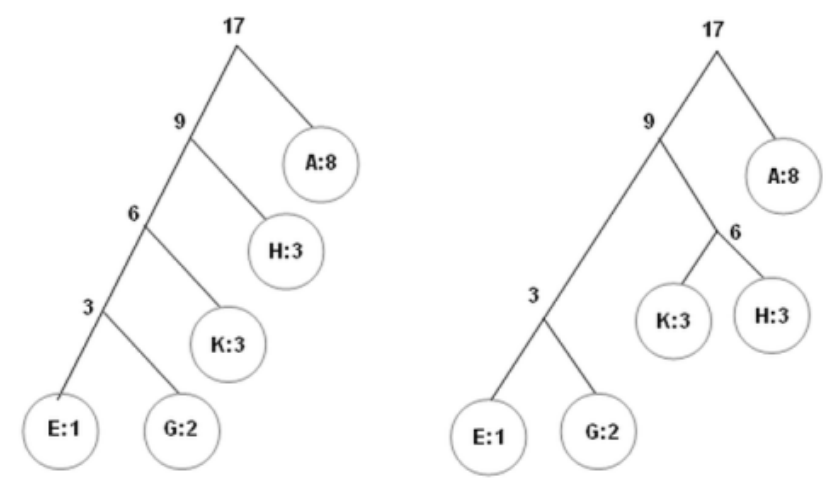

Fig. 7. Example of Huffman trees

To encode the image by means of Huffman's method, the image being analyzed should be converted into a sequence of numbers. It is possible due to defining a histogram for each RGB components. The Huffman coding supplies each of 256 histogram values, encoded with $0-1$ for each RGB component $[9,10]$.

\section{Image recognition}

One of the most difficult steps in image processing and recognition is the detection of objects. To determine what is there on the image requires initial identification of what objects are in the image and what their localization is. The detection of the object is a complex process which requires applying complex algorithms. To facilitate the object detection stage a few steps can be taken. The first step is using line and circle detection algorithms which enable both initial localization 
of the objects and recognizing shapes of the objects on the image. Another step can be colour detection. To satisfy this step first you need to determine the colour which is to be detected and range of this colour. You can achieve this by sampling the colour of a standard object. This process consists of designating the object space followed by designating the average colour and acceptable colour coordinates deviations. In this way the colour range is determined [7, 8].

Another method implies standard matching. This method is generally used when we know the shape of the desired object on the image and it tries to explore what the connection between the template mask and the shape of the given object is. Searching for an object with a desired shape is achieved when the correlation between the standard template mask and the image is defined.

In image processing the standard is a set of features which make up the quantity and quality description of the object. The standard is the features vector:

$$
X=\left[X_{1}, X_{2}, \ldots X_{N}\right]
$$

The standard class is a set of standards characterized by similar feature vectors. Standard classes are marked $\omega_{1}, \omega_{2}, \ldots \omega_{M}$, where index $M$ is the number of the class. Standard recognition in image processing is called classification, whose task is to assign standards to their classes:

$$
X \rightarrow \omega
$$

or space transformation of $X$ feature vectors on the standard class $\Omega$ space. Standards classification consists in assigned $X \rightarrow \omega$ should be correct for a maximum number of standards. Defining such a task depends on how much one knows about the static distribution of a features set. If one cannot define the features distribution or one knows only little about their static distribution the classifier, that is $X \rightarrow \omega$, can be designed by self-learning algorithms based on the set of templates being presented.

\section{Research}

The research was carried out in Matlab system application whose task is to recognize road signs on colourful static images. Having loaded the image the application performs preliminary localization of the object based on the threshold algorithm and standards detection method. For each sign/pixel the RGB values have been determined based on initial research, which means after testing 500 images of selected prohibitory, mandatory, warning and informatory signs. After thresholding to value from 0 to 1 the next step is comparing the obtained shape with the adopted template: circle, triangle or rectangle. The linear Radon transform and the Hough circle transform are implemented on the input image at the same time in order to do the initial localization of the objects on the image. The obtained coordinates of objects, having been localized during the standards detection, are compared to identify the localization of the given objects. 
The correction stage on the input image is followed by removing only scraps containing road signs. In the next step it is resized $128 \times 128$ then it is converted into the sequence of numbers to enable Huffman coding which provides each of 256 histogram values encoded as " 01 " for each RGB component. The values of the signs are saved when the subsequent sign is added to the system in a form of text files transcription.

During sign recognition the analyzed object is compared with all files in the base assigned to one of four groups: prohibitory, mandatory, warning or informatory by means of the chosen distance method. Examples of a running application are presented in Figures 8 and 9.

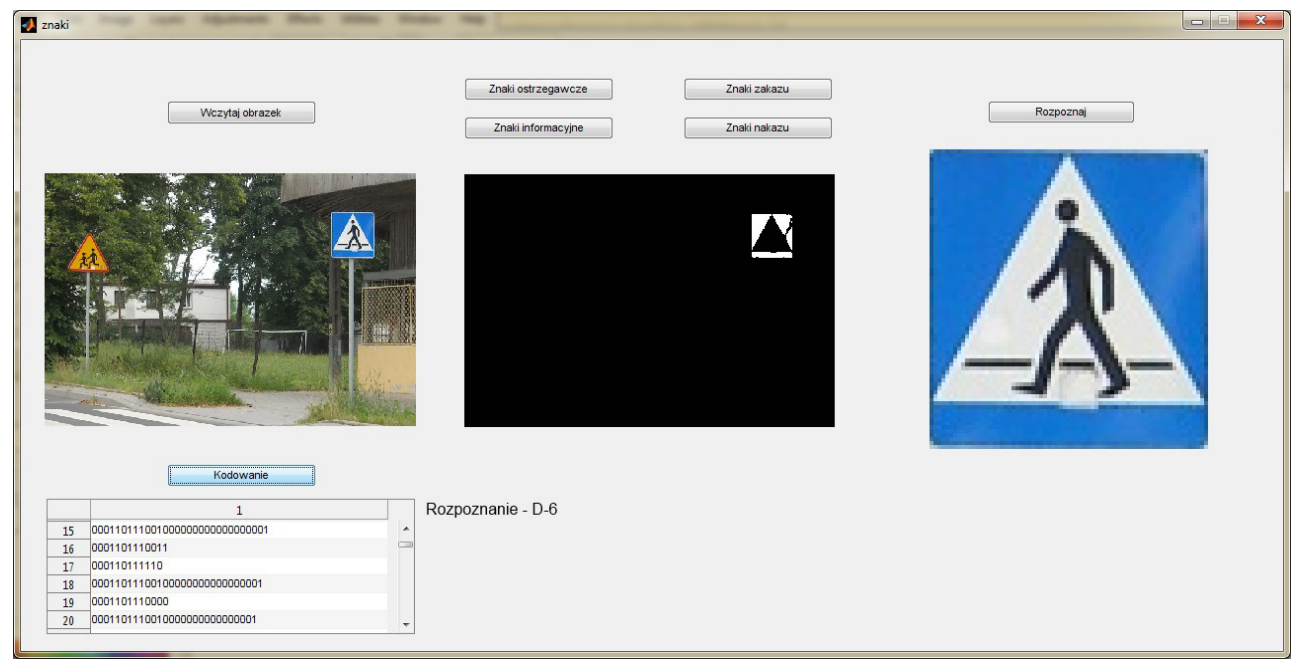

Fig. 8. Example of a running application

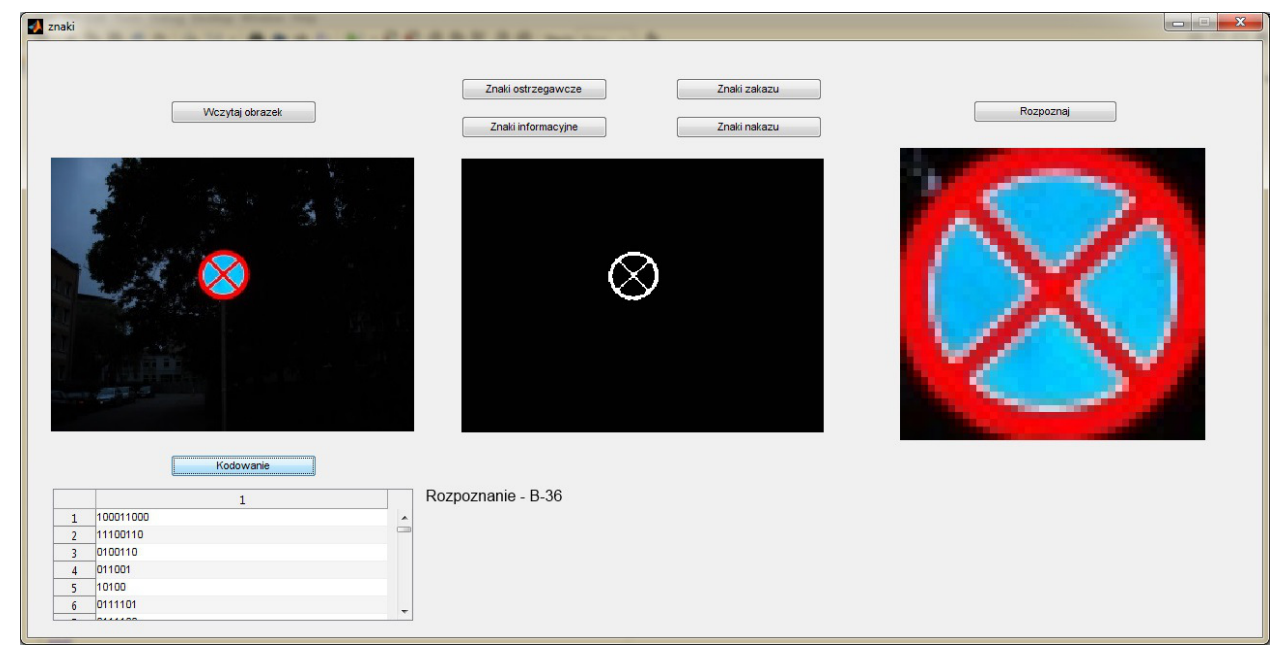

Fig. 9. Example of a running application 
In research 500 images containing road signs were tested taken on a sunny, and rainy day, as well as at night. The image with incorrect initial analysis and detection were also taken into consideration to determine the actual effectiveness of the whole system.

The obtained results are illustrated in the chart below (Table 1 and Figure 10).

Table 1

Results of the road signs recognition depending on the number of signs

\begin{tabular}{|c|c|c|c|}
\hline $\begin{array}{c}\text { Number of test } \\
\text { recognition }\end{array}$ & $\begin{array}{c}\text { Number of road } \\
\text { signs }\end{array}$ & $\begin{array}{c}\text { Number of false } \\
\text { recognitions }\end{array}$ & $\begin{array}{c}\text { The percentage of } \\
\text { false recognitions }\end{array}$ \\
\hline 1 & 100 & 7 & 7 \\
\hline 2 & 200 & 18 & 9 \\
\hline 3 & 300 & 28 & 9.3 \\
\hline 4 & 400 & 46 & 11.5 \\
\hline 5 & 500 & 71 & 14.2 \\
\hline
\end{tabular}

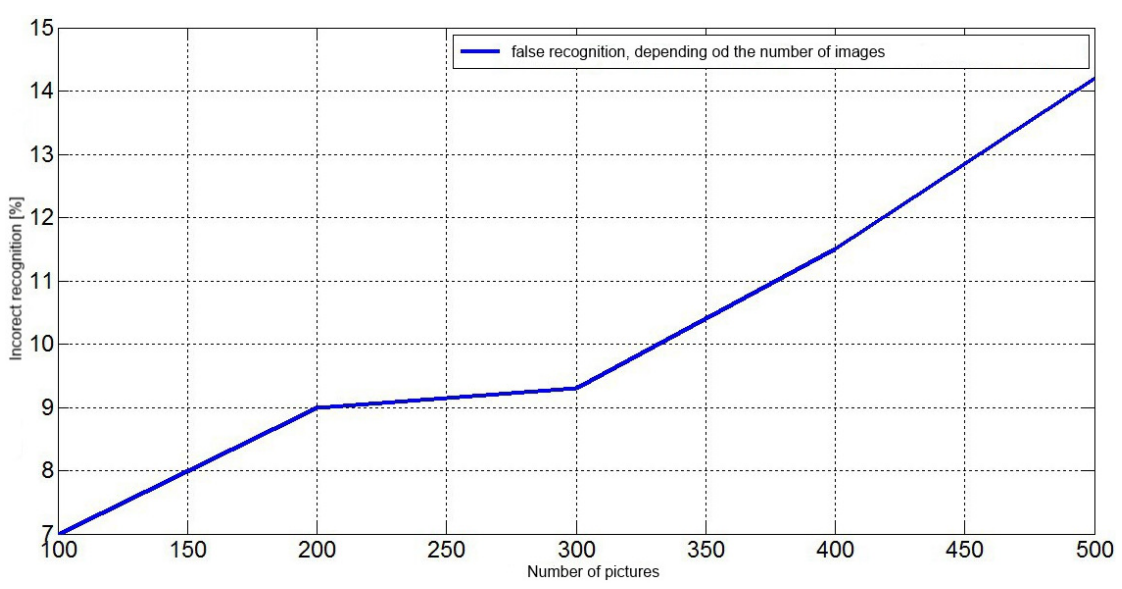

Fig. 10. The dependence of the efficiency of recognition and the number of characters

\section{Conclusion and future work}

The conducted research confirms the rightness of the methods applied and motivates one to continue the undertaken subject. The obtained results are affected by mistakes which are mainly due to imperfect initial analysis and image processing. Reliance on colour images is connected with the problems resulting from the values variability of the RGB components depending on light changes.

Applying only the method of searching for standards which consider only the edges implies that other objects, which are not road signs, can be detected. Compromise and the choice of complementary methods are indispensable. Those features have been applied in the research. 
The application being considered operates only on static images. The further plans will be related with signs recognition on video stream and with applying other encoding type which takes into account the stage of learning (artificial neural networks, hidden Markov models).

\section{References}

[1] Adrjanowicz L., Kubanek M., Bobulski J., Single camera based location estimation with dissimilarity measurement, Proc. of IEEE 6th Int. Conference on Human System Interaction, IEEE Computer Society 2013, 241-246.

[2] Adrjanowicz L., Bobulski J., Kubanek M., State estimation in simultaneous localization and mapping for nonlinear processes with extended Kalman filter, Zeszyty Naukowe Wydziału Elektroniki, Telekomunikacji i Informatyki Politechniki Gdańskiej 2012, 20, 5-10.

[3] http://www.motofakty.pl/artykul/systemy-ulatwiajace-zycie-kierowcy.html

[4] http://www.nissan.pl

[5] http://autokult.p1/2011/05/25/dzialanie-systemu-zapobiegajacego-iekontrolowanej-zmianie-pasa

[6] Wróbel Z., Koprowski R., Praktyka przetwarzania obrazów w programie Matlab, Akademicka Oficyna Wydawnicza EXIT, Warszawa 2004.

[7] Choraś R.S., Komputerowa wizja. Metody interpretacji i identyfikacji obiektów, Akademicka Oficyna Wydawnicza EXIT, Warszawa 2005.

[8] Malina W., Smiatacz M., Cyfrowe przetwarzanie obrazów, Akademicka Oficyna Wydawnicza EXIT, Warszawa 2008.

[9] Skarbek W., Multimedia. Algorytmy i standardy kompresji, Akademicka Oficyna Wydawnicza PLJ, Warszawa 1998.

[10] Sayood K., Kompresja danych wprowadzenie, Wydawnictwo RM, Warszawa 2002. 
